\title{
Anesthesia and Surgery Induce a Functional Decrease Selectively in Excitatory Synaptic Transmission in Mouse Prefrontal Cortex Neurons
}

\section{Yu Matsumoto}

Hyogo College of Medicine

\section{Yuji Fujino}

Osaka University

Hidemasa Furue ( $\square$ hi-furue@hyo-med.ac.jp )

Hyogo College of Medicine

\section{Research Article}

Keywords: Postoperative delirium (POD), anesthesia , surgery, neuropathogenesis, prefrontal cortex (PFC)

Posted Date: December 14th, 2020

DOl: https://doi.org/10.21203/rs.3.rs-123826/v1

License: (c) (i) This work is licensed under a Creative Commons Attribution 4.0 International License.

Read Full License 


\section{Abstract}

Postoperative delirium (POD), a syndrome of confusion and inattention, frequently occurs after anesthesia and surgery. However, the neuropathogenesis of POD remains mostly unknown. The prefrontal cortex (PFC) plays an essential role in cognitive processes. We therefore investigated how anesthesia and surgery induce neurofunctional changes in the PFC, and assessed whether intraoperative administration of dexmedetomidine, an alpha-2 agonist, could prevent the functional changes in the PFC. Laparotomy was performed in mice under isoflurane anesthesia. After a battery of behavioral tests measuring natural behaviors and learning and anxiety levels, PFC neuronal activities were recorded using whole-cell patch-clamp recordings. Effects of intraoperative dexmedetomidine were also examined. In the anesthesia/surgery group, the frequency of excitatory synaptic responses in PFC pyramidal neurons was decreased after the surgery without any changes in the response kinetics. On the other hand, neuronal intrinsic properties and inhibitory synaptic responses were not changed. Intraoperative dexmedetomidine or glutamate receptor antagonists, prevented the excitatory synaptic dysfunction induced by anesthesia and surgery. These findings suggest that anesthesia and surgery induce functional reductions selectively in excitatory synaptic responses in PFC pyramidal neurons, and intraoperative dexmedetomidine inhibits the plastic change in the PFC synaptic transmission.

\section{Introduction}

Perioperative neurocognitive disorders, including delirium (postoperative), have become the most common complications after anesthesia and surgery. ${ }^{1}$ Postoperative delirium (POD), a syndrome of confusion and inattention, occurs in up to $65 \%$ of elderly patients postoperatively. ${ }^{2}$ POD is associated with increased morbidity and mortality, prolonged hospitalization, poor functional recovery, decreased life independence, and long-term decline in cognitive function. ${ }^{3,4}$ However, the pathogenesis of POD is not still fully understood. Although various pathophysiological mechanisms for POD, such as aberrant oxidative cellular metabolism, sympathetic nervous-system stress, perioperative neuroinflammation, and acceleration of Alzheimer's disease, have been proposed, ${ }^{5}$ the mechanisms underlying the neurofunctional changes occurring in POD remain mostly unknown. Since POD presents with cognitive disorders, it is thought to involve functional changes in the CNS such as neurodegeneration and synaptic dysfunction.

The prefrontal cortex (PFC) plays an essential role in cognitive processes such as attention, decisionmaking, and behavioral flexibility. ${ }^{6,7}$ Although the PFC has been reported to be involved in POD, ${ }^{8}$ the postoperative changes in neuronal functions in the PFC require elucidation. Therefore, the primary aim of the current study was to investigate whether anesthesia and surgery induce functional changes in PFC neuronal activities. After a battery of behavior tests to detect delirium-like behavior changes, ${ }^{9}$ we assessed firing properties and subthreshold synaptic transmissions in the PFC using patch-clamp recordings from acute brain slices, and found that the excitatory synaptic transmission was decreased following anesthesia and surgery. Then, based on the fact that there is no approved effective medication 
to prevent or treat POD, our secondary aim was to examine whether intraoperative administration of dexmedetomidine could prevent such functional synaptic decrease in the PFC following anesthesia and surgery. Dexmedetomidine is a highly selective a2 adrenoreceptor agonist that provides anxiolytic, sedative, and modest analgesic effects with minimal respiratory depression, and is widely used in clinical practice. ${ }^{10}$ Although in both preclinical and clinical studies, administration of dexmedetomidine "after" surgery has been reported potentially to prevent occurrence of POD, it is still not approved for use "during" general anesthesia. ${ }^{11,12}$ The present findings may facilitate identification of new underlying mechanisms and preventive measures for POD during anesthesia.

\section{Results}

\section{Anesthesia/surgery did not change the firing pattern of PFC pyramidal neurons postoperatively}

To investigate whether the neuronal activities of PFC pyramidal neurons were altered after anesthesia and surgery, we performed in vitro whole-cell patch-clamp recordings from PFC neurons in brain slice preparations. PFC neurons elicited action potentials in response to $500-\mathrm{ms}$ depolarizing current injections through the recording electrode (Fig. 1A). There were no significant differences in the frequency-current relationship between the control and anesthesia/surgery groups (Fig. 1B). The threshold (control, $-38.5 \pm$ $2.3 \mathrm{mV}, \mathrm{n}=10$; anesthesia/surgery, $-38.5 \pm 2.0 \mathrm{mV}, \mathrm{n}=10 ; p=0.98$ ) and the amplitude (control, $77.7 \pm 1.8$ $\mathrm{mV}, \mathrm{n}=10$; anesthesia/surgery, $78.9 \pm 7.2 \mathrm{mV}, \mathrm{n}=10 ; p=0.71$ ) of the action potentials were not different between the two groups. Resting membrane potentials were not also different (control, $-71.4 \pm 5.6 \mathrm{mV}, \mathrm{n}=$ 10 ; anesthesia/surgery, $-66.8 \pm 4.6 \mathrm{mV}, \mathrm{n}=10 ; p=0.08$ ). These results suggest that anesthesia and surgery do not change the neuronal intrinsic activities of PFC pyramidal neurons.

\section{Anesthesia/surgery decreased presynaptic glutamate- mediated excitatory synaptic transmission in PFC pyramidal neurons}

To assess whether synaptic inputs to PFC pyramidal neurons change after the surgery, we next recorded spontaneous excitatory postsynaptic currents (EPSCs) with whole-cell patch-clamp recordings under voltage-clamp conditions, and compared the frequency and amplitude of spontaneous EPSCs between the two groups. PFC neurons exhibited spontaneous EPSCs. The spontaneous EPSCs frequency in the anesthesia/surgery group was significantly lower (control, $5.5 \pm 2.3 \mathrm{~Hz} ; \mathrm{n}=9$; anesthesia/surgery, $2.8 \pm$ $0.9 \mathrm{~Hz} ; \mathrm{n}=9 ; p=0.006$ ) (Fig. 2). There were no differences in the spontaneous EPSCs amplitude (control, $23.2 \pm 6.6 \mathrm{pA} ; \mathrm{n}=9$; anesthesia/surgery, $21.9 \pm 3.2 \mathrm{pA} ; \mathrm{n}=9 ; p=0.62$ ), the rise time (control, $1.4 \pm 0.3 \mathrm{~ms}$; $\mathrm{n}=9$; anesthesia/surgery, $1.5 \pm 0.2 \mathrm{~ms} ; \mathrm{n}=9 ; p=0.53$ ) and decay time (control, $2.0 \pm 0.6 \mathrm{~ms} ; \mathrm{n}=9$; anesthesia/surgery, $2.0 \pm 0.9 \mathrm{~ms} ; \mathrm{n}=9 ; p=0.98$ ) between the two groups. These results suggest that 
excitatory synaptic input to PFC pyramidal neurons plastically decreases after the surgery without any changes in the synaptic response kinetics.

\section{Anesthesia/surgery did not change inhibitory synaptic transmission in PFC pyramidal neurons postoperatively}

We further compared inhibitory synaptic input to PFC pyramidal neurons by assessing spontaneous inhibitory postsynaptic currents (IPSCs). Spontaneous IPSCs elicited in pyramidal neurons in the PFC were not different between the control and anesthesia/surgery groups. No significant differences were detected in the frequency (control, $7.3 \pm 2.6 \mathrm{~Hz} ; \mathrm{n}=10$; anesthesia/surgery, $8.9 \pm 3.7 \mathrm{~Hz} ; \mathrm{n}=11 ; p=0.29$ ) and amplitude (control, $52.5 \pm 12.0 \mathrm{pA} ; \mathrm{n}=10$; anesthesia/surgery, $46.1 \pm 26.5 \mathrm{pA} ; \mathrm{n}=11 ; p=0.51$ ) of spontaneous IPSCs (Fig. 3). The rise time (control, $2.1 \pm 0.4 \mathrm{~ms} ; \mathrm{n}=10$; anesthesia/surgery, $2.6 \pm 0.6 \mathrm{~ms}$; $\mathrm{n}=11 ; p=0.08$ ) and decay time (control, $4.5 \pm 0.5 \mathrm{~ms} ; \mathrm{n}=10$; anesthesia/surgery, $5.4 \pm 1.5 \mathrm{~ms} ; \mathrm{n}=11 ; p$ $=0.10$ ) of spontaneous IPSCs also did not change. These results suggest that the surgery does not change inhibitory synaptic inputs to PFC pyramidal neurons.

\section{Dexmedetomidine partially alleviated the behavioral impairments induced by Anesthesia/surgery}

We examined behavioral changes in buried food, open field, and $\mathrm{Y}$ maze tests at $24 \mathrm{~h}$ before (baseline), and 6, 9, and $24 \mathrm{~h}$ after isoflurane $2 \mathrm{~h}$ anesthesia (Fig. 4A). In mice that did not receive the anesthesia and surgery (control group), the relative values of the latency to find the buried food $6 \mathrm{~h}$ after the anesthesia to that of baseline did not change $(112.1 \pm 81.8 \%, n=18)$. In mice given a laparotomy during the same period of anesthesia (anesthesia/surgery group), the relative latency significantly increased $6 \mathrm{~h}$ after the anesthesia (322.1 $\pm 408.2 \% ; n=19, p=0.04$ versus the control group, see left graph in Fig. 4B). The anesthesia/surgery group still showed a similar tendency in the relative latencies 9 and $24 \mathrm{~h}$ after the anesthesia, but there were no significant differences between the groups. In mice that underwent a laparotomy under anesthesia with dexmedetomidine at $5 \mu \mathrm{g} / \mathrm{kg}$ (anesthesia/surgery (dexmedetomidine) group, $n=18$ ), the relative latencies to find buried food $6-24 \mathrm{~h}$ after the anesthesia were almost similar to those in the control group, and there were no significant differences between the control and anesthesia/surgery (dexmedetomidine) groups (Fig. 4B). However, there was a significant difference between the latencies $9 \mathrm{~h}$ after the anesthesia in the anesthesia/surgery and anesthesia/surgery (dexmedetomidine) group ( $p=0.006$, see middle graph in Fig. 4B). In the open field test, the total distances travelled in a square chamber were not different between the three groups of mice at $6 \mathrm{~h}$ (control, $74.6 \pm 19.4 \%, n=18$; anesthesia/surgery, $51.1 \pm 19.6 \%, n=19$; and anesthesia/surgery (dexmedetomidine), $53.9 \pm 19.3 \%, \mathrm{n}=18 ; p>0.05$ ), $9 \mathrm{~h}$ (control, $49.1 \pm 26.0 \%, \mathrm{n}=18$; anesthesia/surgery, $43.9 \pm 32.0 \%, \mathrm{n}=19 ;$ anesthesia/surgery (dexmedetomidine), $36.1 \pm 15.2 \%, \mathrm{n}=18 ; p>0.05$ ), and $24 \mathrm{~h}$ (control, $52.7 \pm 21.5 \%, n=18$, anesthesia/surgery, $45.2 \pm 21.4 \%, n=19$; anesthesia/surgery (dexmedetomidine), $43.8 \pm 15.2 \%, \mathrm{n}=18 ; p>0.05$ ) after the anesthesia (Fig. 4C). However, the time spent 
in the center was different between anesthesia/surgery and control groups $6 \mathrm{~h}$ after the anesthesia (control, $70.4 \pm 49.1 \%, \mathrm{n}=18$; anesthesia/surgery, $39.5 \pm 33.4 \%, \mathrm{n}=19 ; p=0.03$ ) (Fig. 4C). No significant differences were observed in the freezing time and latency to the center among the three groups. In the $Y$ maze test, no significant differences were seen among the three groups except between the control and anesthesia/surgery (dexmedetomidine) groups $24 \mathrm{~h}$ after the anesthesia. These results obtained from the battery of behavioral tests suggest that general anesthesia during a laparotomy impaired the natural (buried food test and open field test) behaviors of mice in an acute and fluctuating manner, but intraoperative administration of dexmedetomidine did not change the natural behaviors.

\section{Intraoperative administration of dexmedetomidine or ionotropic glutamate receptor antagonists did not change the frequency of excitatory synaptic transmission in PFC pyramidal neurons}

Lastly, we examined whether dexmedetomidine or the glutamate receptor antagonists MK-801 and CP 465022, could prevent the plastic changes in excitatory synaptic transmission in the PFC after the surgery, and compared the frequency of spontaneous EPSCs among the two groups and the anesthesia/surgery group. As shown in Fig. $5 \mathrm{~A}$ and $\mathrm{B}$, the frequencies of spontaneous EPSCs in both anesthesia/surgery ( $5 \mathrm{mg} / \mathrm{kg}$ dexmedetomidine) and anesthesia/surgery $(0.5 \mathrm{mg} / \mathrm{kg} \mathrm{MK}-801$ and 10 $\mathrm{mg} / \mathrm{kg} \mathrm{CP} 465022)$ groups were significantly greater than that in the anesthesia/surgery group (anesthesia/surgery, $2.7 \pm 1.3 \mathrm{~Hz} ; \mathrm{n}=8$; dexmedetomidine, $7.1 \pm 2.1 \mathrm{~Hz} ; \mathrm{n}=8 ; p<0.01 ; \mathrm{MK}-801$ and $\mathrm{CP}$ $465022,7.3 \pm 4.3 \mathrm{~Hz} ; \mathrm{n}=8$ ). The cumulative distribution of the inter-event interval of spontaneous EPSCs showed a significant leftward shift in the anesthesia/surgery (dexmedetomidine) and anesthesia/surgery (MK-801 and CP 465022) groups ( $p<0.05)$ (Fig. 5C). These results suggest that intraoperative administration of dexmedetomidine or MK-801 and CP 465022, can prevent the synaptic dysfunction induced by anesthesia and surgery.

\section{Discussion}

Here we revealed that a laparotomy under general anesthesia induced a decrease in glutamatergic synaptic inputs to pyramidal neurons of the PFC without changing inhibitory synaptic transmission. Administration of dexmedetomidine or glutamate receptor antagonists during the surgery prevented the plastic change in PFC excitatory synaptic transmission.

We first compared neuronal activities in the PFC between the control and anesthesia/surgery groups. Neuronal electrical activity is primarily dependent on three components: (1) transmitter release from the presynaptic neuron; (2) synaptic responses in the postsynaptic neurons; and (3) firing properties of the postsynaptic neurons. Our electrophysiological results showed that the frequency of spontaneous EPSCs, but not IPSCs, in the PFC decreased in the anesthesia/surgery group, suggesting that presynaptic 
glutamatergic transmitter release to PFC neurons is selectively weakened. However, the amplitude, rise time and decay time of spontaneous EPSCs did not change. These findings suggest that ionotropic glutamate receptors (a-amino-3-hydroxy-5-methylisoxazole-4-propionic acid receptors) expressed in the postsynaptic membrane of the PFC neurons were not altered postoperatively. The firing properties (frequency-current relationship, action potential threshold, and amplitude) of the PFC neurons in addition to resting membrane potentials did not change between the control and anesthesia/surgery groups. Thus, anesthesia/surgery could not impair the postsynaptic neurotransmitter receptor activities, and firing properties, but it modified the excitatory presynaptic inputs to the PFC pyramidal neurons.

Next, we confirmed whether a laparotomy under isoflurane anesthesia induced the behavioral changes associated with delirium. In clinical practice, the confusion assessment method (CAM) is widely used to diagnose the presence of delirium in patients. ${ }^{13}$ The CAM consists of the following four clinical features; acute onset and fluctuating course, inattention, disorganized thinking, and altered level of consciousness. Although it is difficult to detect delirium-like behaviors in animals, Peng et al. developed a battery of behavioral tests in female mice to assess delirium from the viewpoint of CAM. ${ }^{9}$ They reported that an extended latency to find buried food and a reduction in the time spent in the center in the open field test for mice were regarded as the behavioral changes associated with delirium because such parameters depend on the presence and intactness of attention, organized thinking, and consciousness. As reported, in this study anesthesia/surgery also extended the latency to find buried food and decreased the time spent in the center of the open field test, although it did not cause behavioral changes in the $Y$ maze test.

The PFC plays important roles in the functions associated with the symptoms of delirium, including regulation of attention ${ }^{14}$ and organizing thought. ${ }^{15,16} \mathrm{PFC}$ pyramidal neurons are reported to regulate attention and behavior. ${ }^{17}$ Patients with attention deficit hyperactivity disorder (ADHD), whose main symptom is inattention, also exhibited reduced activity of the PFC. ${ }^{18}$ Therefore, behavioral changes such as the delirium-like attention deficits detected in our study may be explained by the synaptic dysfunction (i.e., reduction in the frequency of excitatory synaptic transmission) of PFC pyramidal neurons.

Interestingly, dexmedetomidine partially decreased the buried food latency in the anesthesia/surgery group, and we were not able to detect any differences between the anesthesia/surgery (dexmedetomidine) and control groups in the behavioral tests. These findings suggest that administration of dexmedetomidine during the surgery may inhibit the postoperative changes in behaviors associated with attention, thinking, consciousness, and anxiety.

Then, we investigated whether intraoperative dexmedetomidine could prevent such plastic changes in excitatory synaptic transmission in the PFC after the surgery. Moreover, we also studied whether intraoperative administration of MK-801 and CP 465022, which are antagonists for ionotropic glutamate receptors ( $\mathrm{N}$-methyl-d-aspartate and a-amino-3-hydroxy-5-methylisoxazole-4-propionic acid) could prevent the synaptic dysfunction in the PFC. In the PFC, pyramidal cells are the most abundant neurons (estimated to represent $\sim 80 \%$ or more of the total neuronal population), ${ }^{19}$ and abnormal activations of pyramidal neurons cause excessive glutamate release to induce neurofunctional damage in the CNS. ${ }^{20,21}$ 
Therefore, we hypothesized that an approach specifically targeting inhibition of abnormal activations in neurons should be effective on preventing the synaptic dysfunction after surgery. The abnormal activations mediated by these glutamate receptors is the main pathological process in several neurological diseases such as stroke, epilepsy, and neurodegenerative diseases. Qian et al. showed that systemic administration of MK-801 $(0.5 \mathrm{mg} / \mathrm{kg})$ prevented the brain damage caused by excessive release of glutamate and $\mathrm{N}$-methyl-d-aspartate receptor-related excitotoxicity in stroke. ${ }^{22}$ Menniti et al. also showed that systemic administration of CP $465022(10 \mathrm{mg} / \mathrm{kg})$ potently and effectively inhibited aamino-3-hydroxy-5-methylisoxazole-4-propionic acid receptor-mediated synaptic transmission in the brain. ${ }^{23}$ Moreover, Arias et al. reported that a combination treatment of $\mathrm{N}$-methyl-d-aspartate and aamino-3-hydroxy-5-methylisoxazole-4-propionic acid receptor noncompetitive antagonists produced a synergistic neuroprotective effect in the hippocampus. ${ }^{24}$ In current clinical practice, a level of antinociception during general anesthesia is usually thought to be sufficient when clinical responses, such as body movement and heart rate or blood pressure elevations, no longer occur during surgical stimulation. Opioids are usually used for general anesthesia in conjunction with sedatives to achieve the sufficient level of general anesthesia. However, recent studies have reported that the neuronal activations during surgery in the human brain, particularly in the frontal cortex, cannot be suppressed despite the absence of such clinical responses while propofol and remifentanil are administered together. ${ }^{25}$ Therefore, some supplemental way to inhibit the abnormal activations in the brain could be required to prevent synaptic dysfunction. Actually, our preliminary experiments using in vivo extracellular recordings, showed that neuronal activity in the rat PFC was increased by surgery-like stimuli even under anesthesia condition, and dexmedetomidine were able to suppress its abnormal neuronal activations in the PFC (data not shown). We also found that intraoperative administration of dexmedetomidine or MK-801 and CP 465022 prevented the decrease in glutamate-mediated excitatory synaptic transmission in pyramidal neurons of the PFC. In both preclinical research and clinical studies, administration of dexmedetomidine "after" surgery has been reported to potentially prevent the occurrence of POD. ${ }^{11,12,26}$ Animal studies demonstrated that the mechanism for reduction of cognitive dysfunction was the anti-neuroinflammatory effect of dexmedetomidine. ${ }^{27-29}$ In addition, because dexmedetomidine exerts its effects at both spinal and supraspinal sites to modulate nociceptive input and transmission, and provides analgesia, ${ }^{10}$ it could suppress the neuronal activities elicited by surgery before reaching its nociceptive input to the PFC. Taken together, our study suggests that sequential dexmedetomidine treatment from "during" to "after" surgery may be an effective option for prevention of POD. Moreover, inhibition of glutamate synaptic transmission elicited during surgery might be a novel approach for prevention of POD.

In summary, we found that glutamate-mediated excitatory synaptic transmission in PFC pyramidal neurons decreased "after" anesthesia/surgery, and administration of dexmedetomidine "during " surgery could prevent the synaptic change of PFC neurons.

\section{Methods}

\section{Animals}


Female animals are known to exhibit less anxiety than male animals in behavioral assessments performed in an open space. ${ }^{30}$ We first performed open field test and compared time spent in the center of the open field test (see below) between C57BL/ 6 male and female mice. In male mice, the time spent in the center of the 2 nd trial was decreased to less than half of it ( $48 \pm 12.5 \%$ of the first trail, $n=6)$. That of female mice was not largely changed $(74.6 \%$ of the first trial, see Results), suggesting that anxiety in male mice is not stable even without anesthesia and surgery. To detect behavioral changes including anxiety following anesthesia and surgery, a recent study developed a battery of behavioral analyses using female animals. ${ }^{9}$ In this study, we therefore used female animals (C57BL/ 6 mice (SLC, Hamamatsu, Japan)) to detect behavioral and neuronal changes following anesthesia and surgery. The animals were housed in cages with food and water available ad libitum. The room was maintained at $20^{\circ} \mathrm{C}$ with a 12-h light/dark cycle. All animal studies were reviewed and approved by the Institutional Animal Care and Use Committee of Hyogo College of Medicine, and were performed in accordance with the institutional guidelines for animal experiments and carried out in accordance with the ARRIVE guidelines 2.0. Every effort was made to reduce the number of animals used.

\section{Behavioral tests and electrophysiological recordings from mice after anesthesia and surgery}

Mice were randomly divided into three groups: control, Anesthesia/surgery, and anesthesia/surgery (dexmedetomidine). Mice in the anesthesia/surgery and anesthesia/surgery (dexmedetomidine) groups received a laparotomy under general anesthesia (see below). In the two groups, a battery of behavior tests was conducted 6, 9, and $24 \mathrm{~h}$ after surgery. The same battery of behavioral tests was also performed $24 \mathrm{~h}$ before the surgery to obtain baseline behavioral data. In the control group, mice were placed in their home cages, and the battery of behavioral tests was administered at the same four time points as in other two groups. We performed in vitro whole-cell patch-clamp recordings for PFC neurons in acute brain slice preparations from the mice of all three groups within 14 days after anesthesia and surgery.

\section{Anesthesia and surgery}

Laparotomy was performed in 8-10-week-old mice under isoflurane anesthesia, similar to previous studies. ${ }^{9,31}$ Anesthesia was induced and maintained using $1.5 \%$ isoflurane in $100 \% \mathrm{O}_{2}$ in a transparent acrylic box for $15 \mathrm{~min}$, after which anesthesia was maintained via a cone-shaped mask over the nose. A longitudinal incision was made from the xiphoid to pubic symphysis $(0.5 \mathrm{~cm})$ on the skin, abdominal muscles, and peritoneum, after which the incision was sutured with 5-0 vicryl thread. The procedure required approximately $10 \mathrm{~min}$. The mouse was then transferred back into the box, and was under anesthesia for $2 \mathrm{~h}$ in total. EMLA cream ${ }^{\circledR}$ (2.5\% lidocaine and $2.5 \%$ propitocaine, Astra Pharmaceuticals) was applied onto the incision at the end of the procedure and at 8-h intervals for one day. Dexmedetomidine hydrochloride at a dose of $5 \mu \mathrm{g} / \mathrm{kg}$ (intraperitoneal) and a mixture of MK-801, a 
noncompetitive $\mathrm{N}$-methyl-d-aspartate receptor antagonist at a dose of $0.5 \mathrm{mg} / \mathrm{kg}$ (intraperitoneal), and CP 465022, a noncompetitive a-amino-3-hydroxy-5-methylisoxazole-4-propionic acid receptor antagonist at a dose of $10 \mathrm{mg} / \mathrm{kg}$ (subcutaneously) were administered $20 \mathrm{~min}$ before the surgery.

\section{Buried food test}

The buried food test was performed as described in previous studies with modifications. ${ }^{32,33}$ We used ten 45-mg pieces of a purified pellet of F-0021 J (BrainScience idea Co., Ltd.). The mice were habituated to the testing room for $1 \mathrm{~h}$. The test cages $(30 \times 5 \times 15 \mathrm{~cm})$ were prepared with clean bedding (height, $3 \mathrm{~cm})$ and a round-shaped case (diameter, $2 \mathrm{~cm})$ containing the pellets. The case was buried in the bedding $(0.5 \mathrm{~cm}$ below to the surface), and the location of the case in the test cage was changed at random. The mouse was placed in the center of the test cage, and the time to start eating was measured. If the mouse did not find the pellet in $5 \mathrm{~min}$, we ended the trial and considered $300 \mathrm{~s}$ as the latency period. We used a new test cage, bedding, and gloves for each mouse.

\section{Open field test}

Open field test was performed as described in previous studies with modifications. ${ }^{9,34}$ The open field apparatus was a white plexiglass square box $(40 \times 40 \times 40 \mathrm{~cm})$. The mouse was gently placed at the center of the box under dim lighting and was allowed to move freely for $5 \mathrm{~min}$. The mouse in the box was monitored by a video camera, and the behavioral trajectory was analyzed with Any-maze behavior tracking system software (Stoelting Co., Wood Dale, IL). The total distance traveled in the box, the time spent in the center of the open field, the freezing time, and the latency to reach the center of the open field at the first attempt were analyzed. The floor of the open field was cleaned with $70 \%$ ethanol solution after every test.

\section{Y maze test}

$\mathrm{Y}$ maze test was performed as described in previous studies, with modifications. ${ }^{9,35,36}$ The $Y$ maze apparatus was made of black-colored plexiglass. The apparatus consisted of three arms $(30 \times 5 \times 15 \mathrm{~cm})$ with an angle of $120^{\circ}$ between each arm. The three arms were the start arm, in which the mouse starts to explore (always open); the novel arm, which is blocked at the first trial, but opened at the second trial; and the other arm (always open). The start arm and the other arm were randomly designed to avoid the spatial memory error. The $Y$ maze test consisted of 2 trials separated by an inter-trial interval (ITI). The first trial (training) was performed for $10 \mathrm{~min}$ and allowed the mouse to explore 2 arms (start arm and other arm) of the maze, with the novel arm being blocked. After an ITI of $1 \mathrm{~h}$, the second trial (retention) was performed. For the second trial, the mouse was placed back in the maze in the same starting arm, with free access to all three arms for $5 \mathrm{~min}$. The maze was placed in a quiet and illustrated room. The behavior was recorded by a video camera mounted above the maze, and the number of entries and the 
time spent in each arm were assessed with the Any-maze behavior tracking system software. The time spent in and entries into the novel arm were used as an indication of spatial recognition memory. Each of the arms of the $Y$ maze was cleaned with $70 \%$ ethanol solution before testing.

\section{In vitro whole-cell patch-clamp recording}

Mice that underwent the behavioral tests were rapidly decapitated after cervical dislocation, and the brain was quickly removed and submerged in a cold artificial cerebrospinal fluid (ACSF) solution containing (in mM) $124 \mathrm{NaCl}, 2.5 \mathrm{KCl}, 2 \mathrm{CaCl}_{2}, 1 \mathrm{MgSO}_{4}, 25 \mathrm{NaHCO}_{3}, 1 \mathrm{NaH}_{2} \mathrm{PO}_{4}$, and 10 glucose equilibrated with $95 \%$ $\mathrm{O}_{2}-5 \% \mathrm{CO}_{2}$. Coronal brain slices $(300 \mu \mathrm{m})$ at the level of the PFC were prepared using a vibratome. ${ }^{37-39}$ The brain slices were transferred to a submerged recovery chamber with the ACSF solution at room temperature for at least $1 \mathrm{~h}$. Individual slices were then put into a recording chamber in which the ACSF solution was continuously perfused at $32-33^{\circ} \mathrm{C}$. Whole-cell patch-clamp recordings were made from

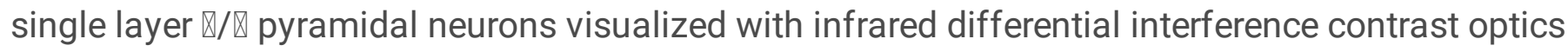
(BX50WI, Olympus, Tokyo, Japan) ${ }^{39}$. The patch pipettes (3-5 M $\Omega$ ) were filled with a potassium-based intracellular solution containing (in $\mathrm{mM}$ ): K-glucose $135, \mathrm{CaCl}_{2} 0.5, \mathrm{MgCl}_{2} 2$, EGTA 5, ATP-Mg 5, and HEPES 5; pH: 7.2 for recordings of EPSCs and membrane potentials, or a cesium-based intracellular solution containing (in $\mathrm{mM}$ ): $\mathrm{Cs}_{2} \mathrm{SO}_{4} 110$, TEA-Cl 5, $\mathrm{CaCl}_{2} 0.5, \mathrm{MgCl}_{2}$ 2, EGTA 5, ATP-Mg 5 and HEPES$\mathrm{CsOH} 5 ; \mathrm{pH}: 7.2$ for recordings of IPSCs. EPSCs and IPSCs were recorded under voltage-clamp conditions at a holding potential of $-70 \mathrm{mV}$ and $0 \mathrm{mV}$, respectively. Membrane potentials and action potentials (APs) were recorded under current-clamp conditions. APs were evoked by $500-\mathrm{ms}$ depolarizing pulses from 0 to 150 pA with 30-pA steps through the recording electrode from a membrane potential of $-70 \mathrm{mV}$. The access resistance (15-30 M $\Omega$ ) was monitored throughout the experiment. Data were discarded if the resistance changed by more than $20 \%$ during the recording. The recording signals were amplified with a patch-clamp amplifier (MultiClamp 700A; Molecular Devices, Sunnyvale, CA, USA), low-pass filtered at 5 $\mathrm{kHz}$, digitized with an analog-to-digital converter (Digidata 1321 A; Molecular Devices), and stored on a personal computer using a data acquisition program (pCLAMP version 12.3; Molecular Devices) with a sampling frequency of $10-20 \mathrm{kHz}$. Synaptic events were analyzed using a software (Minianalysis version 6.0.7; Synaptosoft, Fort Lee, NJ, USA). Only cells exhibiting a stable resting membrane potential (less than $-50 \mathrm{mV}$ ) were used in this study. The following criteria were used to measure action potential threshold and amplitude. The first action potential evoked by the minimum injection current was used for all measurements. Action potential threshold is the voltage where a sharp upward rise of the depolarizing phase of the action potential occurs, while action potential amplitude is the difference in voltage between the threshold and its peak amplitude

\section{Drugs}

Dexmedetomidine was obtained from Maruishi Pharmaceutical Co., LTD. (Osaka, Japan) and was dissolved in saline (1 $\mu \mathrm{g} / \mathrm{mL})$. MK-801 and CP 465022 were obtained from Tocris Bioscience (Bristol, 
England) and dissolved in saline at $25 \mu \mathrm{g} / \mathrm{mL}$ and $2 \mathrm{mg} / \mathrm{mL}$, respectively.

\section{Statistical analysis}

All numerical data are shown as mean \pm SD. Statistical analyses were performed with $\mathrm{EZR}^{40}$ (Saitama Medical Center, Jichi Medical University, Saitama, Japan), which is a graphical user interface for R (The R Foundation for Statistical Computing, Vienna, Austria). More precisely, it is a modified version of $\mathrm{R}$ commander designed to perform statistical functions frequently used in biostatistics. Student's unpaired $t$ tests was used to compare the firing properties, the parameters of EPSCs and IPSCs between control and anesthesia/surgery group. Student's paired $t$ tests was used to compare the firing frequency between before and after stimuli. Two-way repeated analysis of variance (ANOVA) followed by post hoc Bonferroni test was used to compare the frequency-current relationship of action potentials and the cumulative distributions of inter-event interval of spontaneous EPSCs. The Kruskal-Wallis test followed by post hoc Steel-Dwass was used to analyze the behavioral test results of the three groups (control, anesthesia/surgery, anesthesia/surgery (dexmedetomidine)) and the frequency of spontaneous EPSCs for the three groups (anesthesia/surgery, anesthesia/surgery (MK-801 and CP465022), anesthesia/surgery (dexmedetomidine)). In all cases, $p<0.05$ was considered to be statically significant. " $n$ " refers to the number of animals or neurons.

\section{Declarations}

\section{Acknowledgments}

We would like to thank Dr. K Takao for their helpful suggestions and behavioral technical support, and $\mathrm{K}$ Koga and T Date for their helpful advices. We are deeply grateful to the care and management of laboratory animals by the staff of Center for Comparative Medicine in Hyogo College of Medicine.

\section{Author Contributions}

YM performed experiments and YM, YF and HF analyzed data. HF supervised the experiments. All authors contributed to experimental design and writing and/or editing the manuscript. All authors have read and approved the final version of this manuscript.

\section{Additional Information}

The authors declare no competing interests.

\section{Funding}


This work was supported by JSPS KAKENHI Grant Numbers JP17H04114, JP20H04043, and institutional Hyogo Innovative Challenge grant.

\section{References}

1. Evered, L. et al. Recommendations for the nomenclature of cognitive change associated with anaesthesia and surgery-2018. British journal of anaesthesia 121, 1005-1012, doi:10.1016/j.bja.2017.11.087 (2018).

2. Rudolph, J. L. \& Marcantonio, E. R. Review articles: postoperative delirium: acute change with longterm implications. Anesth Analg 112, 1202-1211, doi:10.1213/ANE.0b013e3182147f6d (2011).

3. Inouye, S. K., Westendorp, R. G. \& Saczynski, J. S. Delirium in elderly people. Lancet (London, England) 383, 911-922, doi:10.1016/s0140-6736(13)60688-1 (2014).

4. Abelha, F. J. et al. Outcome and quality of life in patients with postoperative delirium during an ICU stay following major surgery. Critical care (London, England) 17, R257, doi:10.1186/cc13084 (2013).

5. Mahanna-Gabrielli, E. et al. State of the clinical science of perioperative brain health: report from the American Society of Anesthesiologists Brain Health Initiative Summit 2018. British journal of anaesthesia 123, 464-478, doi:10.1016/j.bja.2019.07.004 (2019).

6. Fuster, J. M. The prefrontal cortex-an update: time is of the essence. Neuron 30, 319-333, doi:10.1016/s0896-6273(01)00285-9 (2001).

7. Miller, E. K. The prefrontal cortex and cognitive control. Nat Rev Neurosci 1, 59-65, doi:10.1038/35036228 (2000).

8. Cascella, M., Muzio, M. R., Bimonte, S., Cuomo, A. \& Jakobsson, J. G. Postoperative delirium and postoperative cognitive dysfunction: updates in pathophysiology, potential translational approaches to clinical practice and further research perspectives. Minerva anestesiologica 84, 246-260, doi:10.23736/s0375-9393.17.12146-2 (2018).

9. Peng, M. et al. Battery of behavioral tests in mice to study postoperative delirium. Scientific reports 6 , 29874, doi:10.1038/srep29874 (2016).

10. Nguyen, V., Tiemann, D., Park, E. \& Salehi, A. Alpha-2 Agonists. Anesthesiol Clin 35, 233-245, doi:10.1016/j.anclin.2017.01.009 (2017).

11. Su, X. et al. Dexmedetomidine for prevention of delirium in elderly patients after non-cardiac surgery: a randomised, double-blind, placebo-controlled trial. Lancet (London, England) 388, 1893-1902, doi:10.1016/s0140-6736(16)30580-3 (2016).

12. Zhu, Y. S., Xiong, Y. F., Luo, F. Q. \& Min, J. Dexmedetomidine protects rats from postoperative cognitive dysfunction via regulating the GABA(B) R-mediated cAMP-PKA-CREB signaling pathway. Neuropathology: official journal of the Japanese Society of Neuropathology 39, 30-38, doi:10.1111/neup.12530 (2019).

13. Inouye, S. K. et al. Clarifying confusion: the confusion assessment method. A new method for detection of delirium. Annals of internal medicine 113, 941-948, doi:10.7326/0003-4819-113-12-941 
(1990).

14. Buschman, T. J. \& Miller, E. K. Top-down versus bottom-up control of attention in the prefrontal and posterior parietal cortices. Science (New York, N.Y.) 315, 1860-1862, doi:10.1126/science.1138071 (2007).

15. Aron, A. R., Robbins, T. W. \& Poldrack, R. A. Inhibition and the right inferior frontal cortex. Trends in cognitive sciences 8, 170-177, doi:10.1016/j.tics.2004.02.010 (2004).

16. Best, M., Williams, J. M. \& Coccaro, E. F. Evidence for a dysfunctional prefrontal circuit in patients with an impulsive aggressive disorder. Proceedings of the National Academy of Sciences of the United States of America 99, 8448-8453, doi:10.1073/pnas.112604099 (2002).

17. Arnsten, A. F. \& Pliszka, S. R. Catecholamine influences on prefrontal cortical function: relevance to treatment of attention deficit/hyperactivity disorder and related disorders. Pharmacol Biochem Behav 99, 211-216, doi:10.1016/j.pbb.2011.01.020 (2011).

18. Sharma, A. \& Couture, J. A review of the pathophysiology, etiology, and treatment of attention-deficit hyperactivity disorder (ADHD). The Annals of pharmacotherapy 48, 209-225, doi:10.1177/1060028013510699 (2014).

19. DeFelipe, J. \& Farinas, I. The pyramidal neuron of the cerebral cortex: morphological and chemical characteristics of the synaptic inputs. Progress in neurobiology 39, 563-607, doi:10.1016/03010082(92)90015-7 (1992).

20. Levite, M. Glutamate receptor antibodies in neurological diseases. Journal of neural transmission (Vienna, Austria: 1996) 121, 1029-1075, doi:10.1007/s00702-014-1193-3 (2014).

21. Meldrum, B. S. Glutamate as a neurotransmitter in the brain: review of physiology and pathology. The Journal of nutrition 130, 1007s-1015s, doi:10.1093/jn/130.4.1007S (2000).

22. Qian, Y., Tang, X., Guan, T., Li, Y. \& Sun, H. Neuroprotection by Combined Administration with Maslinic Acid, a Natural Product from Olea europaea, and MK-801 in the Cerebral Ischemia Model. Molecules (Basel, Switzerland) 21, doi:10.3390/molecules21081093 (2016).

23. Menniti, F. S. et al. CP-465,022, a selective noncompetitive AMPA receptor antagonist, blocks AMPA receptors but is not neuroprotective in vivo. Stroke 34, 171-176, doi:10.1161/01.str.0000048216.90221.9c (2003).

24. Arias, R. L., Tasse, J. R. \& Bowlby, M. R. Neuroprotective interaction effects of NMDA and AMPA receptor antagonists in an in vitro model of cerebral ischemia. Brain Res 816, 299-308, doi:10.1016/s0006-8993(98)01051-8 (1999).

25. Lichtner, G. et al. Nociceptive activation in spinal cord and brain persists during deep general anaesthesia. British journal of anaesthesia 121, 291-302, doi:10.1016/j.bja.2018.03.031 (2018).

26. Djaiani, G. et al. Dexmedetomidine versus Propofol Sedation Reduces Delirium after Cardiac Surgery: A Randomized Controlled Trial. Anesthesiology 124, 362-368, doi:10.1097/aln.0000000000000951 (2016).

27. Xiong, B., Shi, Q. \& Fang, H. Dexmedetomidine alleviates postoperative cognitive dysfunction by inhibiting neuron excitation in aged rats. American journal of translational research $\mathbf{8}, 70-80$ (2016). 
28. Zhu, Y. J., Peng, K., Meng, X. W. \& Ji, F. H. Attenuation of neuroinflammation by dexmedetomidine is associated with activation of a cholinergic anti-inflammatory pathway in a rat tibial fracture model. Brain Res 1644, 1-8, doi:10.1016/j.brainres.2016.04.074 (2016).

29. Peng, M., Wang, Y. L., Wang, C. Y. \& Chen, C. Dexmedetomidine attenuates lipopolysaccharideinduced proinflammatory response in primary microglia. The Journal of surgical research 179, e219225, doi:10.1016/j.jss.2012.05.047 (2013).

30. Kokras, N. \& Dalla, C. Sex differences in animal models of psychiatric disorders. Br J Pharmacol 171, 4595-4619, doi:10.1111/bph.12710 (2014).

31. Lu, Y. et al. Surgery/Anesthesia disturbs mitochondrial fission/fusion dynamics in the brain of aged mice with postoperative delirium. Aging 12, 844-865, doi:10.18632/aging.102659 (2020).

32. Lehmkuhl, A. M., Dirr, E. R. \& Fleming, S. M. Olfactory assays for mouse models of neurodegenerative disease. Journal of visualized experiments: JoVE, e51804, doi:10.3791/51804 (2014).

33. Nathan, B. P., Yost, J., Litherland, M. T., Struble, R. G. \& Switzer, P. V. Olfactory function in apoE knockout mice. Behav Brain Res 150, 1-7, doi:10.1016/s0166-4328(03)00219-5 (2004).

34. Zhao, H. et al. Upregulation of Beta4 subunit of BKCa channels in the anterior cingulate cortex contributes to mechanical allodynia associated anxiety-like behaviors. Molecular brain 13, 22 , doi:10.1186/s13041-020-0555-z (2020).

35. Chen, Y. et al. Anxiety- and depressive-like behaviors in olfactory deficient Cnga2 knockout mice. Behav Brain Res 275, 219-224, doi:10.1016/j.bbr.2014.08.042 (2014).

36. Rayatnia, F. et al. Nitric oxide involvement in consolidation, but not retrieval phase of cognitive performance enhanced by atorvastatin in mice. European journal of pharmacology $666,122-130$, doi:10.1016/j.ejphar.2011.05.017 (2011).

37. Koga, K. et al. Coexistence of two forms of LTP in ACC provides a synaptic mechanism for the interactions between anxiety and chronic pain. Neuron $85,377-389$, doi:10.1016/j.neuron.2014.12.021 (2015).

38. Li, X. Y. et al. Alleviating neuropathic pain hypersensitivity by inhibiting PKMzeta in the anterior cingulate cortex. Science (New York, N.Y.) 330, 1400-1404, doi:10.1126/science.1191792 (2010).

39. Matsumoto, Y., Fujino, Y. \& Furue, H. Anti-nociceptive and anxiolytic effects of systemic flupirtine and its direct inhibitory actions on in vivo neuronal mechanical sensory responses in the adult rat anterior cingulate cortex. Biochem Biophys Res Commun, doi:10.1016/j.bbrc.2020.07.129 (2020).

40. Kanda, Y. Investigation of the freely available easy-to-use software 'EZR' for medical statistics. Bone marrow transplantation 48, 452-458, doi:10.1038/bmt.2012.244 (2013).

\section{Figures}


A

Control

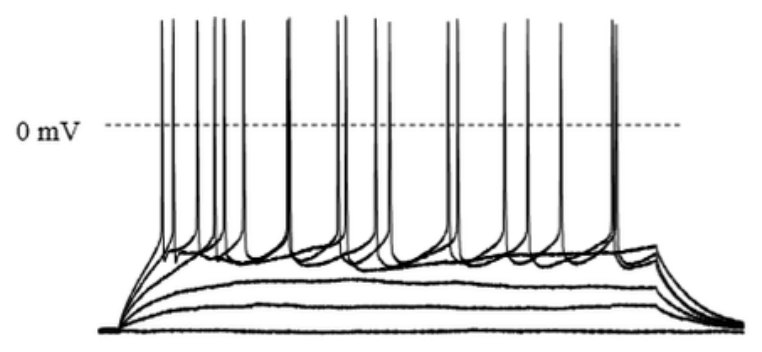

Anesthesia/Surgery

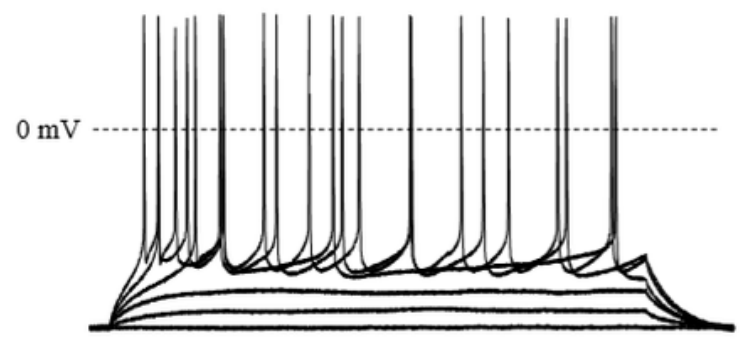

$20 \mathrm{mV}$

$0.1 \mathrm{~ms}$

B

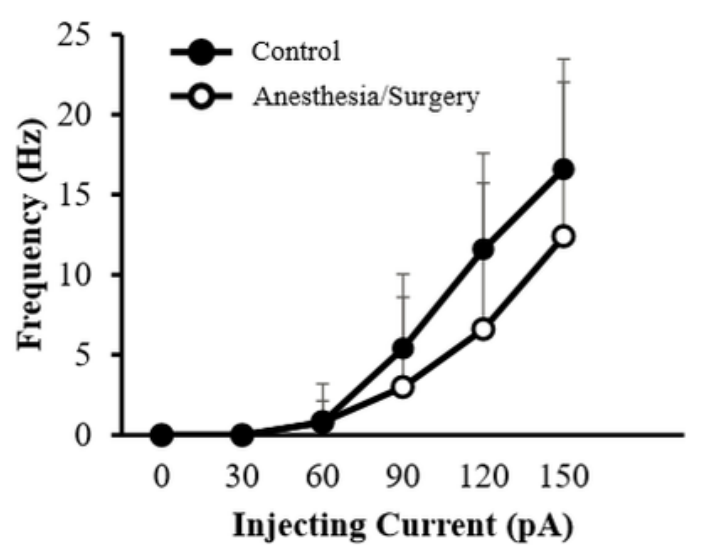

\section{Figure 1}

Anesthesia and surgery did not change the firing properties of pyramidal neurons in the PFC (A) Examples of firing properties of PFC pyramidal neurons in control and anesthesia/surgery groups. In response to current injections applied through the recording pipette, pyramidal neurons in both groups showed a tonic firing. (B) Relationships between the frequency of action potentials and amplitudes of the 
current injected. There were no significant differences in the frequency-current relationships between control and anesthesia/surgery groups (control, $n=10$; anesthesia/surgery, $n=10 ; p=0.28$ ).

A

Control

Anesthesia/Surgery
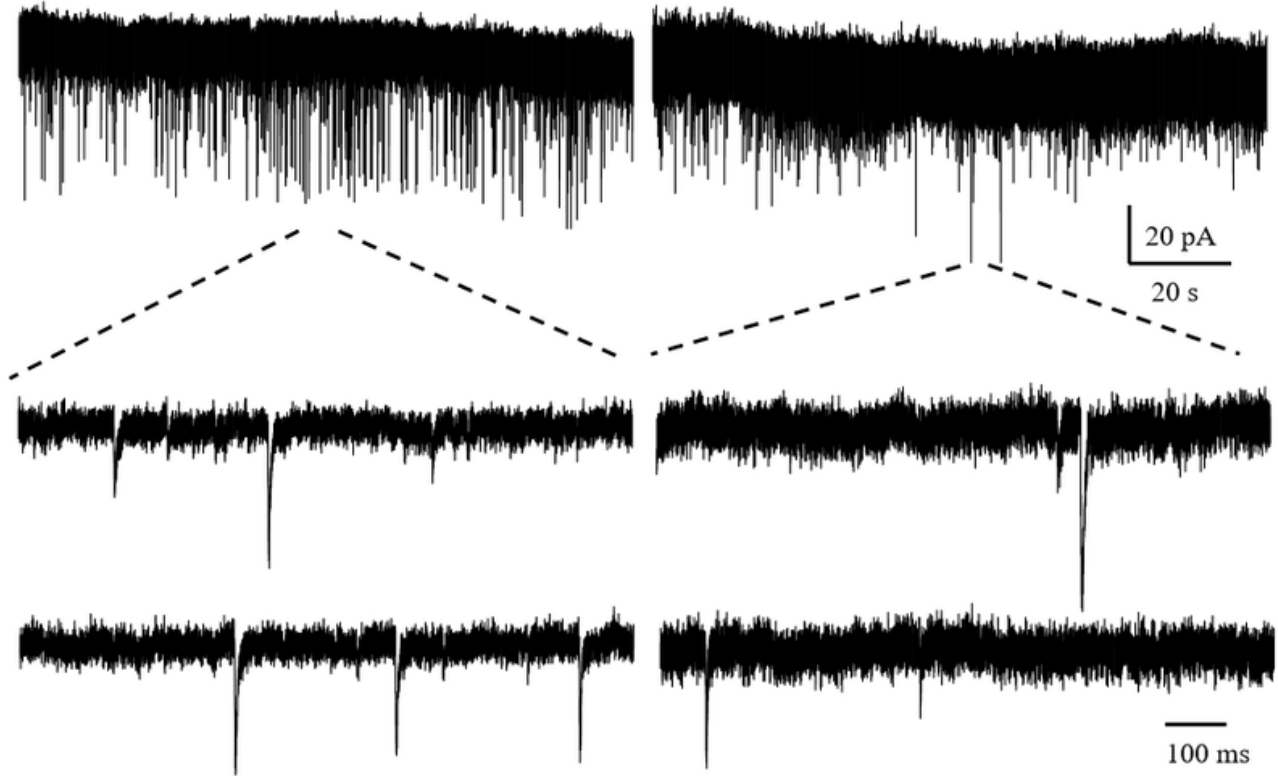

B
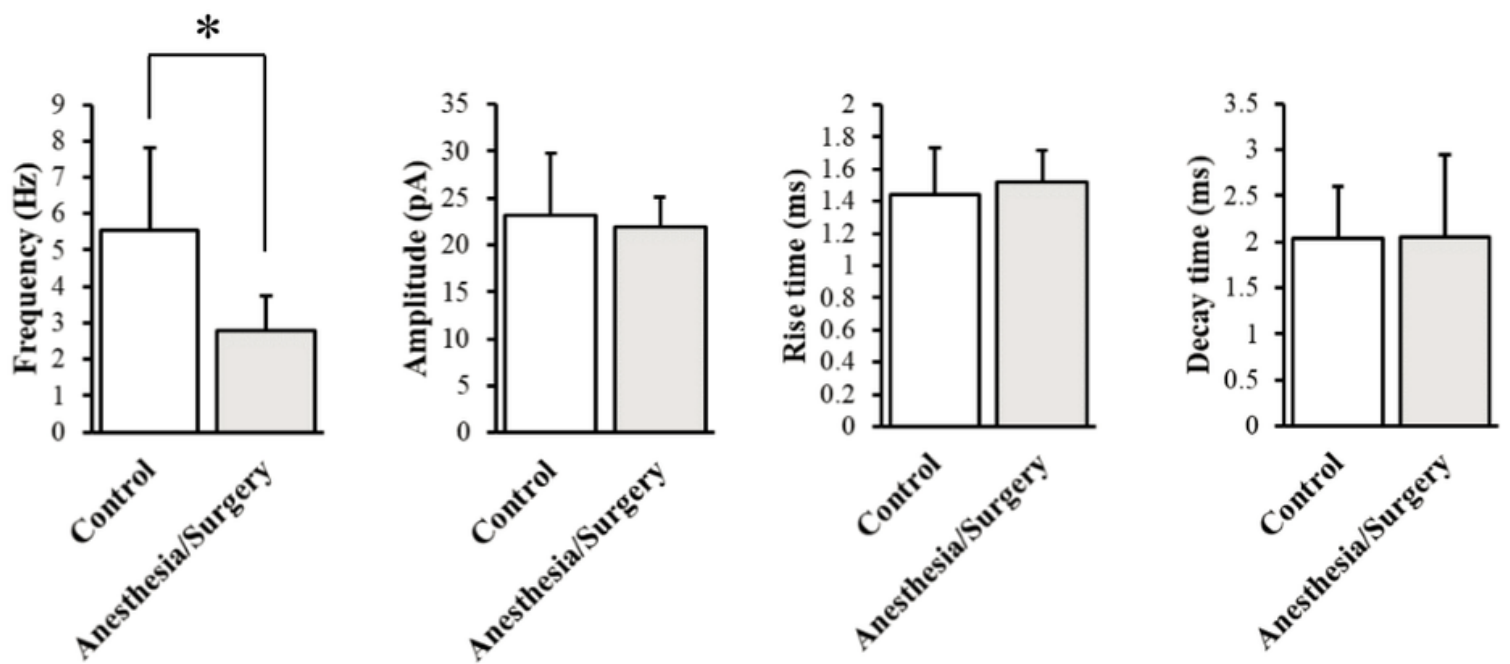

Figure 2

Anesthesia and surgery decreased glutamate-mediated excitatory synaptic transmission in PFC pyramidal neurons. (A) Examples of spontaneous EPSCs recorded from PFC neurons under a voltageclamp condition at a holding potential of $-70 \mathrm{mV}$ in control and anesthesia/surgery groups. Lower two traces are shown in an expanded timescale. (B) Summary showing the frequency, amplitude, rise time, and decay time of spontaneous EPSCs. The frequency of spontaneous EPSCs in the anesthesia/surgery 
group was significantly lower than that in control group (control, $n=9$; anesthesia/surgery, $n=9$; * $p=$ 0.006).
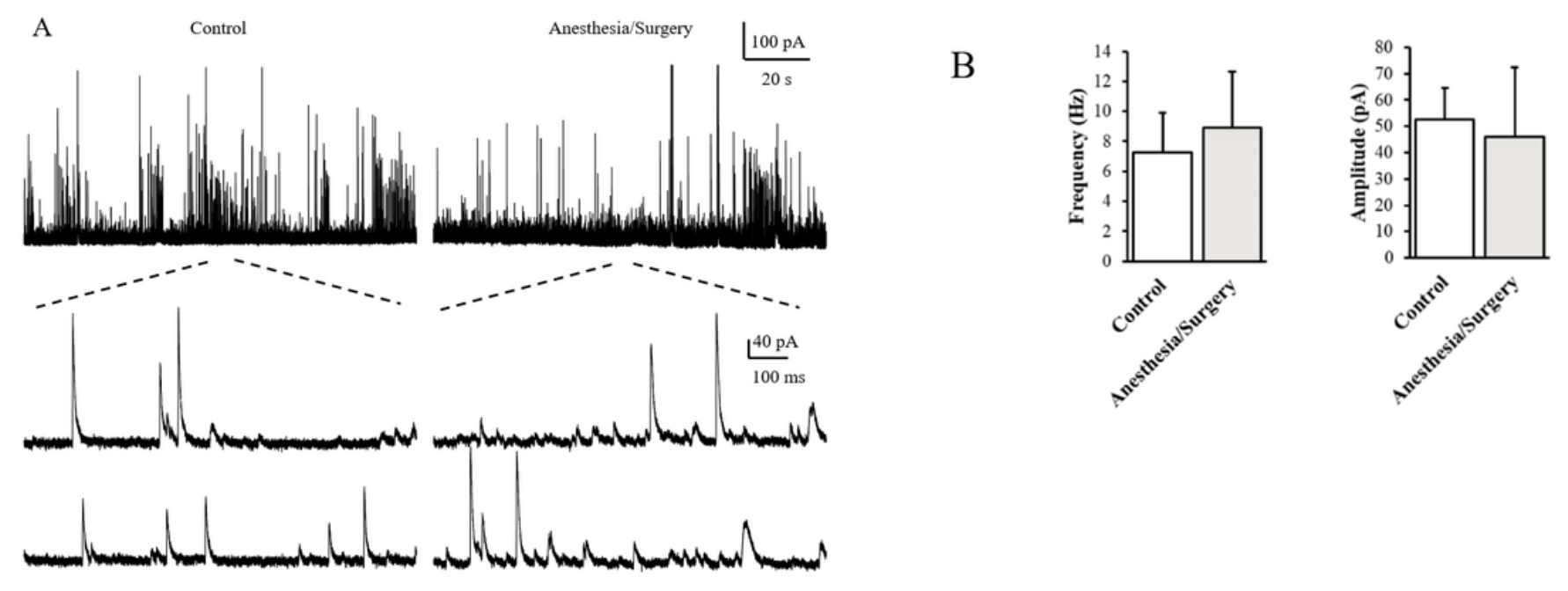

Figure 3

Anesthesia and surgery did not change inhibitory synaptic transmission in PFC pyramidal neurons. (A) Examples of spontaneous IPSCs recorded from PFC neurons under a voltage-clamp condition at a holding potential of $0 \mathrm{mV}$ in control and anesthesia/surgery groups. Lower two traces are shown in an expanded timescale. (B) Summary showing no differences in the frequency, amplitude of spontaneous IPSCs between the two groups (control, $n=9$; anesthesia/surgery, $n=9 ; p>0.05$ ). 
A

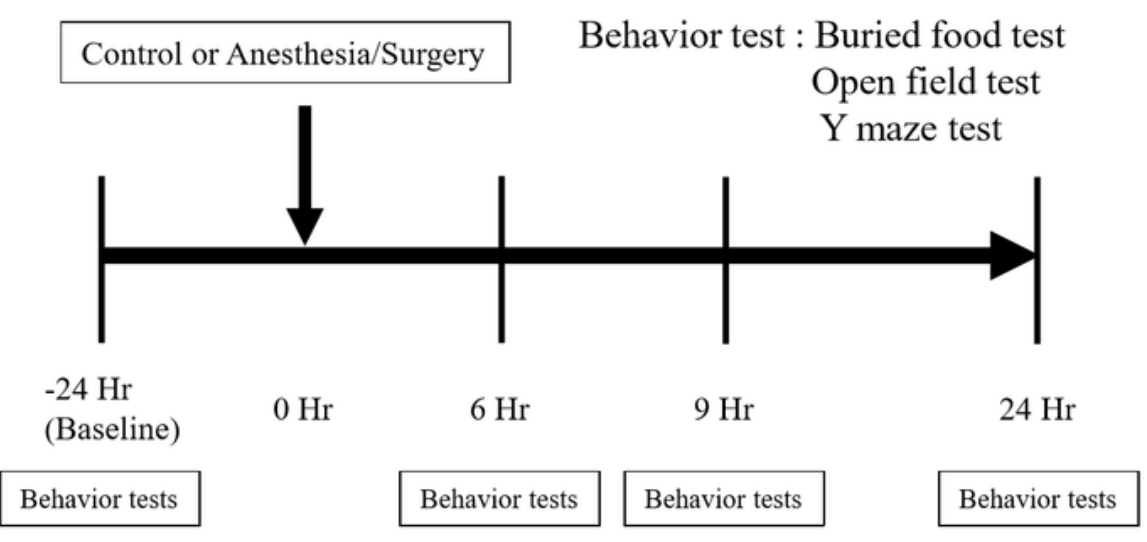

B Buried food test
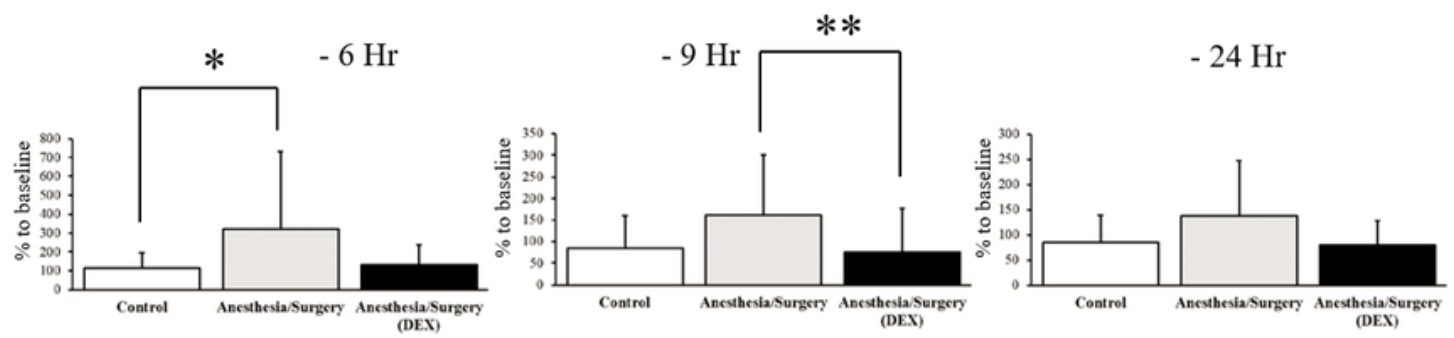

C Open field test

Total distance $-6 \mathrm{Hr}$

$-9 \mathrm{Hr}$

$-24 \mathrm{Hr}$
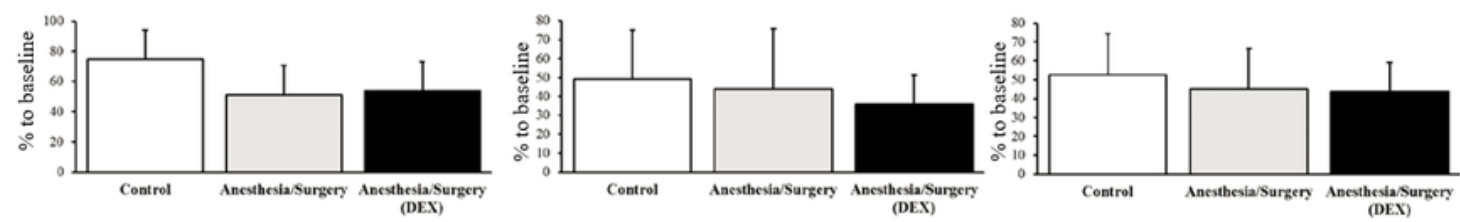

The time spent in the center $-6 \mathrm{Hr}$

$-9 \mathrm{Hr}$

$-24 \mathrm{Hr}$
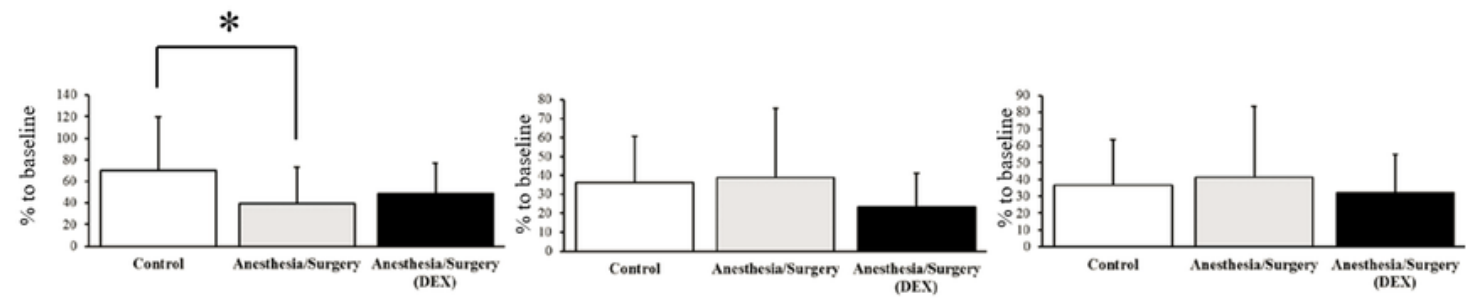

\section{Figure 4}

Action of intraoperative administration of dexmedetomidine on the natural behavioral alterations induced by anesthesia and surgery (A) Diagram of the experimental design. (B) Summary showing the relative values of the latencies to find buried food 6,9 , and $24 \mathrm{~h}$ after anesthesia and surgery to the baseline latency obtained from the same mice $24 \mathrm{~h}$ before anesthesia and surgery. The relative latency $6 \mathrm{~h}$ after anesthesia and surgery was higher in the anesthesia/surgery group than in the control group. There were 
no differences between anesthesia/surgery (dexmedetomidine) $(5 \mu \mathrm{g} / \mathrm{kg}$ ) and control groups, and the latency $9 \mathrm{~h}$ after anesthesia and surgery was lower in the anesthesia/surgery (dexmedetomidine) group in comparison with the anesthesia/surgery group. Control group, $n=18$; anesthesia/surgery group, $n=$ 19; anesthesia/surgery (dexmedetomidine) group, $n=18$; $* p<0.05$, $* * p<0.01$. (C) Summary showing relative values of the total distance of the open field test after anesthesia and surgery to the baseline total distance obtained from the same mice $24 \mathrm{~h}$ before anesthesia and surgery (upper three graphs). Anesthesia/surgery group did not show differences in the total distance in the open field in comparison with the control group at 6, 9, and $24 \mathrm{~h}$ after anesthesia and surgery. Lower three graphs showing that anesthesia/surgery group showed lower relative values of time spent in the center of the open field in compared with the control group at 6 , but not at 9 or $24 \mathrm{~h}$ after anesthesia and surgery. Control group, $\mathrm{n}=$ 18; anesthesia/surgery group, $n=19$; anesthesia/surgery (dexmedetomidine) group, $n=18,{ }^{*} p<0.05$.

A

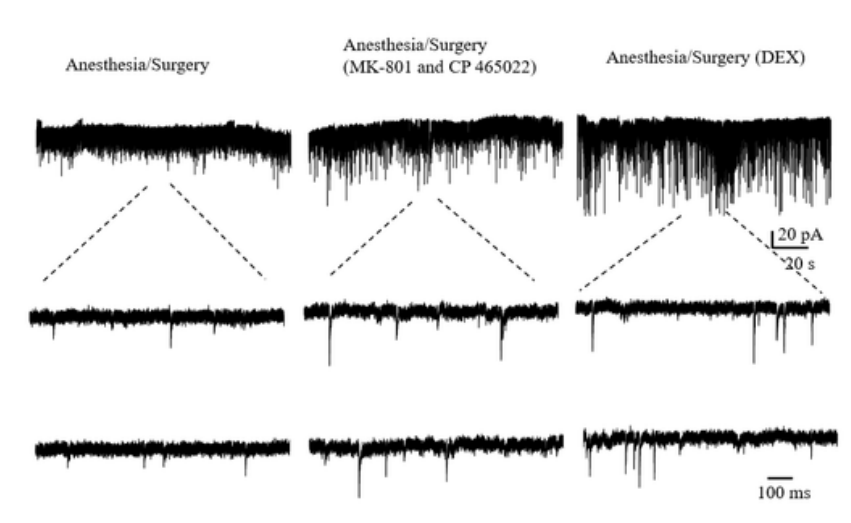

B

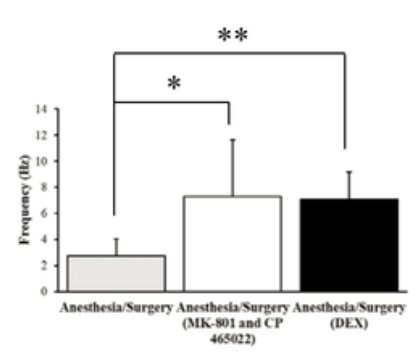

C

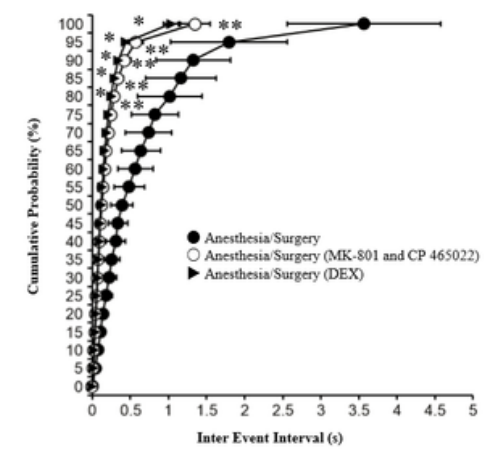

\section{Figure 5}

Intraoperative administration of dexmedetomidine or glutamate receptor antagonists prevented the functional decrease in glutamate-mediated excitatory synaptic transmission in PFC pyramidal neurons.

(A) Examples of spontaneous EPSCs recorded from PFC neurons in anesthesia/surgery, anesthesia/surgery (0.5 mg/kg MK-801 and $10 \mathrm{mg} / \mathrm{kg}$ CP 465022) and anesthesia/surgery (5 $\mu \mathrm{g} / \mathrm{kg}$ dexmedetomidine) groups. Lower two traces are shown in an expanded timescale. (B) Summary showing the frequency of spontaneous EPSCs in the three groups. The frequencies of spontaneous EPSCs both in anesthesia/surgery (MK-801 and CP 465022) and anesthesia/surgery (dexmedetomidine) groups were higher than those in the anesthesia/surgery group (anesthesia/surgery, $n=8$; anesthesia/surgery (MK801 and CP 465022), $n=8$; anesthesia/surgery (dexmedetomidine), $n=8$; $*<<0.05$, ** $p<0.01$ ). (C) Cumulative distributions of spontaneous EPSCs inter event-interval in the anesthesia/surgery, anesthesia/surgery (MK-801 and CP 465022), and anesthesia/surgery (dexmedetomidine). There were significant leftward shifts in the distributions in the anesthesia/surgery (MK801 and CP465022), and anesthesia/surgery (dexmedetomidine) groups (anesthesia/surgery, $n=8$; anesthesia/surgery (MK801 and CP 465022); ${ }^{\star *} \mathrm{p}<0.05$ v.s. anesthesia/surgery, $\mathrm{n}=8$; anesthesia/surgery (dexmedetomidine), $\mathrm{n}=8$; ${ }^{*} \mathrm{p}<0.05$ v.s. anesthesia/surgery). 